\title{
Involvement of DDX6 gene in radio- and chemoresistance in glioblastoma
}

\author{
YU JIN CHO ${ }^{1}$, WONYOUNG KANG ${ }^{7}$, SUNG HEON KIM $^{4}$, JASON K. SA ${ }^{3}$, NAYOUNG KIM ${ }^{3}$, \\ PATRICK J. PADDISON ${ }^{5}$, MISUK KIM ${ }^{6}$, KYEUNG MIN JOO ${ }^{4}$, YOUNG-IL HWANG ${ }^{1}$ and DO-HYUN NAM ${ }^{2,3}$ \\ ${ }^{1}$ Department of Anatomy, Seoul National University College of Medicine, Seoul; ${ }^{2}$ Department of Neurosurgery, \\ Samsung Medical Center, Sungkyunkwan University School of Medicine, Seoul; ${ }^{3}$ Department of Health Sciences \\ and Technology, SAIHST, Sungkyunkwan University, Seoul; ${ }^{4}$ Department of Anatomy and Cell Biology, \\ Sungkyunkwan University of Medicine, Suwon, Seoul, Republic of Korea; ${ }^{5}$ Department of Human Biology Division, \\ Fred Hutchinson Cancer Research Center, Seattle, WA, USA; ${ }^{6}$ Institute for Refractory Cancer Research, \\ Samsung Medical Center; ${ }^{7}$ Samsung Biomedical Research Institute, Samsung Medical Center, Seoul, Republic of Korea
}

Received October 5, 2015; Accepted November 6, 2015

DOI: 10.3892/ijo.2016.3328

\begin{abstract}
CCRT (concomitant chemotherapy and radiation therapy) is often used for glioblastoma multiforme (GBM) treatment after surgical therapy, however, patients treated with CCRT undergo poor prognosis due to development of treatment resistant recurrence. Many studies have been performed to overcome these problems and to discover genes influencing treatment resistance. To discover potential genes inducing CCRT resistance in GBM, we used whole genome screening by infecting shRNA pool in patient-derived cell. The cells infected $\sim 8,000$ shRNAs were implanted in mouse brain and treated RT/TMZ as in CCRT treated patients. We found DDX6 as the candidate gene for treatment resistance after screening and establishing DDX6 knock down cells for functional validation. Using these cells, we confirmed tumor associated ability of DDX6 in vitro and in vivo. Although proliferation improvement was not found, decreased DDX6 influenced upregulated clonogenic ability and resistant response against radiation treatment in vivo and in vitro. Taken together, we suggest that DDX6 discovered by using whole genome screening was responsible for radio- and chemoresistance in GBM.
\end{abstract}

Correspondence to: Professor Young-il Hwang, Department of Anatomy, Seoul National University College of Medicine, Seoul, Republic of Korea

E-mail: hyi830@snu.ac.kr

Professor Do-Hyun Nam, Department of Neurosurgery, Samsung Medical Center, Sungkyunkwan University School of Medicine, Seoul, Republic of Korea

E-mail:nsnam@skku.edu

Key words: glioblastoma multiforme, concomitant chemotherapy and radiation therapy, radiation, temozolomide, resistance, xenograft model, patient-derived cell

\section{Introduction}

Standard therapy, CCRT (concomitant chemotherapy and radiation therapy), is the most effective method and the only curable therapy for glioblastoma multiforme (GBM) except surgery $(1,2)$. This method is composed of radiation and temozolomide (TMZ) treatment for GBM patients. Radiation has been an effective therapeutic method for various cancer types for a long time; however, repeated radiation treatment often induces radioresistance $(3,4)$. Although TMZ is the only active medicine available for GBM patients, it is not beneficial for every patient. TMZ response varies in each patient because of MGMT [O-(6)-methylguanine-DNA methyltransferase] enzyme expression, which induces DNA repairment of the damaged DNA after TMZ treatment. Methylation status of MGMT promoter is important in enzyme expression (5). Patients with unmethylated MGMT promoter show resistance to TMZ treatment and long-term treatment also induces resistance to $\mathrm{TMZ}$ as seen in radioresistance $(6,7)$. To overcome such obstacle, many groups have studied radiation/TMZ resistance mechanism and developed substitute treatment methods (8-12).

shRNA (short hairpin RNA) library screening method for targeting genome has been used for discovering new therapeutic targets or tumor associated genes due to the shRNA ability to induce deprived gene functions $(13,14)$. ShRNA induction experiments are conducted to identify specific genes that are associated with drug treatment, metastasis, and transcriptional activity. Several tumor suppressor genes have been discovered by using such screening method (14-16). In this study, we discovered the radiation/TMZ resistance related gene DDX6, using in vivo shRNA screening and demonstrated that suppression of DDX6 induces resistance to radiation/TMZ treatment. DDX6 is a RNA helicase and regulates mRNA translation and storage in P-bodies. Recently, Chen et al reported CNOT1 (CCR4-NOT complex 1) interacts with DDX6 for mRNA decapping in human cells and CNOT1 complex regulates mRNA translation in breast 
cancer and acute lymphoblastic leukemia (17). Although mRNA regulation and indirection with other tumorassociated complex of DDX6 have been reported, direct tumor-associated actions still remain unknown. Herein, we present for the first time the resistant mechanism of DDX6 to antitumor treatment.

\section{Materials and methods}

Patient-derived cell and sphere culture. According to the Institutional Review Boards, specimens were obtained from glioblastoma patients after surgery. Patient-derived cells were cultured in Neurobasal-A medium (Thermo Fisher Scientific, Waltham, MA, USA) supplemented with $\mathrm{N} 2$ and B27 supplements (0.5x respectively, Gibco) and human recombinant bFGF (20 $\mathrm{ng} / \mathrm{ml}, \mathrm{R} \& \mathrm{D}$ systems, MN, USA) and EGF (20 ng/ml, R\&D Systems) $(18,19)$. All used patient-derived cells were maintained under 20 passages in vitro.

Viral production. 293FT cells (Invitrogen) were transfected with target vectors and viral package vectors (pCMV-PAX2 and pCMV-VSVG) by using CalPhos ${ }^{\mathrm{TM}}$ (Clontech Laboratories, Inc., Mountain View, CA, USA) according to the manufacturer's protocol. There are target vectors for pGIPZ for shRNA screening and DDX6 shRNA vectors (MISSON ${ }^{\circledR}$ shRNA, Sigma, St. Louis, MO, USA) for gene validation. All vectors have puromycin selection markers. The manufactured lentiviral supernatants were filtered through a $0.45 \mu \mathrm{m}$ filter to remove cell debris and centrifuge at $20,000 \times \mathrm{g}$ for $2 \mathrm{~h}$ at $4^{\circ} \mathrm{C}$ to concentrate the virus supernatant.

Pooled shRNA screening. shRNA pool was generated using pGIPZ system (GE System, CO, USA) and information of pGIPZ vector was based on the website (http://dharmacon. gelifesciences.com). Target genes for shRNA were chosen randomly. The 827 patient-derived cells were infected with the shRNA library and selected by puromycin $(0.5 \mu \mathrm{g} /$ $\mathrm{ml}$ ) for 3 days. After 3 days, selected cells were sorted by fluorescence-activated cell sorter (FACSAria ${ }^{\mathrm{TM}}$, BD Biosciences, Franklin Lakes, NJ, USA) for mouse implantation. The control cells (before mouse implantation) and mass were harvested at the mouse survival end point for genetic analysis. shRNA barcodes were PCR-recovered from genomic samples and analyzed through next generation sequencing (Illumina High-Seq 2000, San Diego, CA, USA). shRNA level were normalized to its whole population and relative alteration of shRNA expression were measured. shRNAs presented in 2 or more replicates were selected for next experiment.

Generation of candidate gene knockdown cells. Five shRNAs specifically targeting the candidate gene DDX6, was used to reduce gene expression. The sequences of shRNAs were derived from the DDX6 coding region, but the control shRNA did not depress the DDX6 expression (pLKO, Sigma). Transduced cells were harvested for western blotting and successfully depressed DDX6 clone was selected. Among five shRNAs, only one shRNA was accepted, and used for functional validation.
Orthotopic xenograft model. Six-week-old male BALB/c nude mice (Orient Bio Inc., Seoul, South Korea) were used for pooled shRNA screening and gene validation. Patientderived GBM cells (5x10 /mouse) were intracranially injected in mouse brain by stereotactic instrument $(\mathrm{AP}+0.5 \mathrm{~mm}$, $\mathrm{ML}+1.7 \mathrm{~mm}$, DV $-3.2 \mathrm{~mm}$ from the bregma). Radiation or temozolomide (TMZ) treatment were performed at time two thirds of the control group median survival. Each mouse was sacrificed when unusual conditions (cachexia, lethargy and seizures) or $20 \%$ body weight loss were observed. For survival analysis, 5 mice were used per groups. All mouse experiments were performed according to the Association for Assessment and Accreditation of Laboratory Animal Care-accredited guidelines of Samsung Medical Institute's Animal Use and Care Committee (Permit number: K-B2-035).

Western blot assay. Cell lysis was performed in RIPA buffer supplemented with protease inhibitor cocktail tablets (Roche, Basel, Switzerland). Total proteins (10 $\mu \mathrm{g} /$ lane) were separated in SDS-PAGE gel and transferred to PVDF membranes (Millipore). The membranes were blocked for $2 \mathrm{~h}$ in room temperature by $5-10 \%$ skim milk solution and were incubated with primary antibodies (rabbit anti-DDX6 (Abcam, Cambridge, UK) and mouse anti- $\beta$-catenin (Santa Cruz, Biotechnology, Inc., Dallas, TX, USA) overnight at $4^{\circ} \mathrm{C}$. After washing with TBST (Tris-Buffered Saline, $0.1 \%$ Tween-20), the membranes were incubated with HRP-conjugated secondary antibodies for $1 \mathrm{~h}$ at $4^{\circ} \mathrm{C}$. Development of membrane was performed using the chemiluminescence method (ECL, GE Healthcare, Pittsburgh, PA, USA).

Cell proliferation and viability test. Cell proliferation and viability were performed using EZ-cytox cell viability kit (DAEIL Lab, South Korea) according to the manufacturer's protocol. For cell proliferation, $0.5-1 \times 10^{3}$ cells were seeded per well in 96-well plate and each sample was in triplicate. After 3-6 days, Ez-cytox was added into each well and incubated for $2 \mathrm{~h}$. The absorbance at $450 \mathrm{~nm}$ was measured for incubated plates. Cell viability were also performed according to the manufacturer's protocol with treatment schedule. Radiation or temozolomide (TMZ) treatment was administered within $6 \mathrm{~h}$ of seeding.

Sphere limiting dilution assay (SLDA) and sphere counting. Sphere limiting dilution assay (SLDA) was performed according to published method (20). Briefly, each of the cells was plated at densities range from 500 to 2 cells in a 96-well plate with 6 replicates for each dilution and evaluated after several days in culture. We scored each well for the absence (-) or presence $(+)$ of sphere growth to determine the fraction of negative wells. The plot shows percentage for the fraction of non-responding wells (y-axis) versus plating density (x-axis). In addition, sphere counting was performed when each of the cells had similar clonogenic ability in SLDA.

Establishment of radiation/TMZ resistant cell lines. We established radiation/TMZ resistant cell lines by modifying pre-published method $(21,22)$. Briefly, radiation or TMZ treatment was performed for each cell at lethal dose and these cells were incubated several days to recover their population. 
A

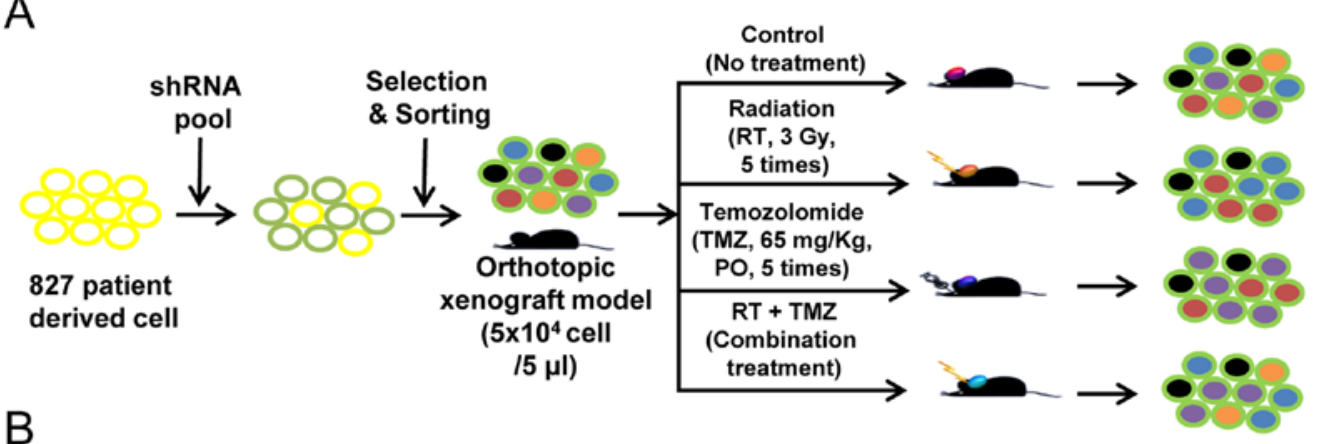

B
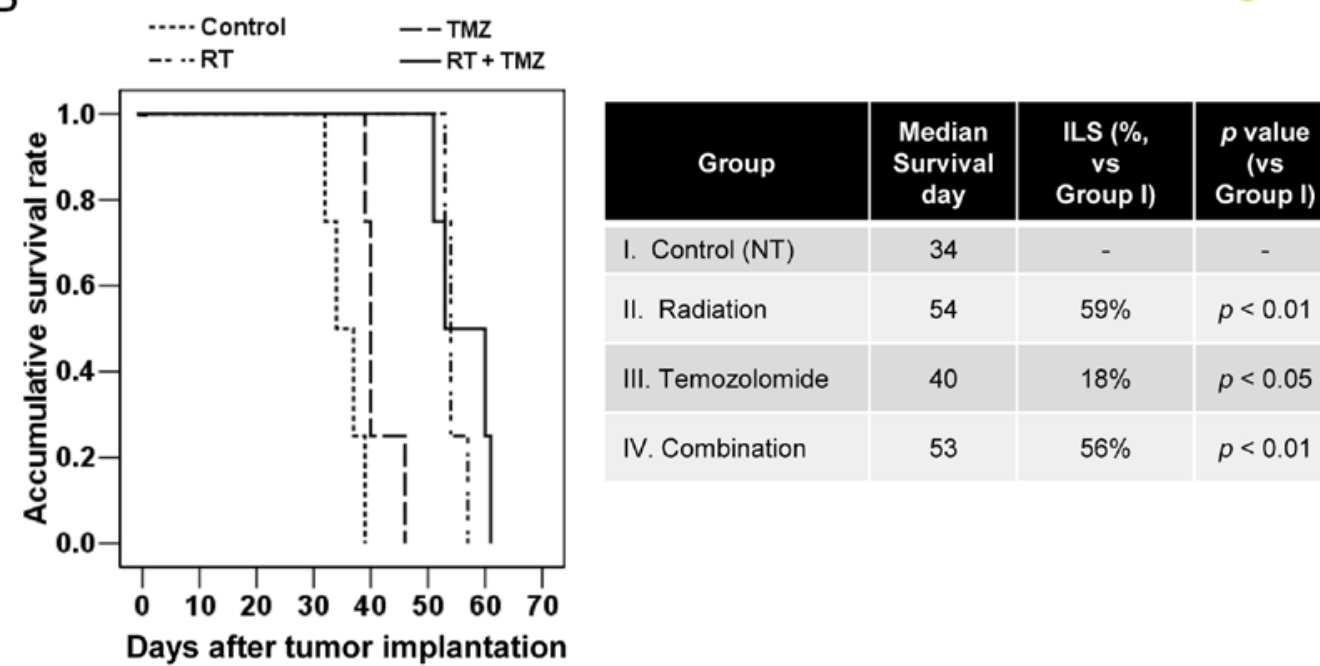

C

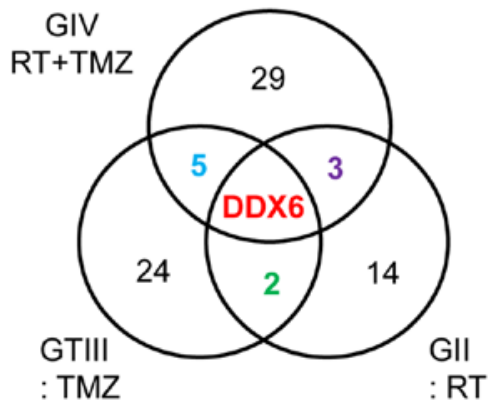

\section{GII and GIII $\quad$ GII and GIV $\quad$ GIII and GIV}

\begin{tabular}{|l|c|c|c|}
\hline I. Control (NT) & 34 & - & - \\
\hline II. Radiation & 54 & $59 \%$ & $p<0.01$ \\
\hline III. Temozolomide & 40 & $18 \%$ & $p<0.05$ \\
\hline IV. Combination & 53 & $56 \%$ & $p<0.01$ \\
\hline
\end{tabular}

\begin{tabular}{|c||c|c|}
\hline & & DDX6 \\
\hline DDX6 & DDX6 & DDAH1 \\
\hline MYD88 & BTG3 & MGC16025 \\
SLC10A5 & PRMT7 & RFX7 \\
& PRTG & TGFB1 \\
& & ZNF649 \\
\hline
\end{tabular}

Figure 1. Pooled shRNA screening for CCRT resistance associated gene using patient-derived cell xenograft model. (A) shRNA screening experiment scheme using the 827 sample orthotopic xenograft model. (B) Survival rate of the 827 tumor-bearing mice. ILS was significantly changed by radiation and temozolomide treatment. (C) Highly expressed shRNAs targeted DDX6 after treatment in all treatment groups.

For competent resistant cell lines, repeated treatments were conducted several times and these cells were confirmed by toxicity test using EZ-cytox cell viability kit.

Statistics. All statistical analysis was conducted using Student's t-test to determine the significance of results $(\mathrm{P}<0.05, \mathrm{P}<0.01$, $\mathrm{P}<0.001)$. Overall survival curves were plotted according to the Kaplan-Meier method.

\section{Results}

Pooled shRNA screening for CCRT resistance. Screening using a shRNA library was used to identify CCRT resistancerelated genes. Patient-derived cells treated with the various shRNAs were implanted in mouse brains. The mice received a standard treatment of GBM, radiation and/or TMZ, and the remaining tumor mass was harvested from each mouse at the survival end-point for analysis of shRNA expression change (Fig. 1A). Survival increased in all treatment groups as compared with control group. However, survival gain in the combination therapy group (GIV, ILS $=56 \%$ ) was similar to the radiation therapy group (GII, ILS $=59 \%$ ) (Fig. 1B). These data resulted from different responses of the 827 cell samples to radiation therapy (RT) and TMZ. Since the 827 samples were sensitive to RT and already resistant to TMZ, no combination-related survival gain was evident. The next experiments were done to demonstrate these characteristics in vitro (Figs. 5 and 6). Next generation sequencing analysis of the remaining tumor mass revealed shRNAs with increased expression, which enabled selection of the targeted genes in commonly expressing genes of three references to ensure that the genetic change noted in the 827 cells was 
A

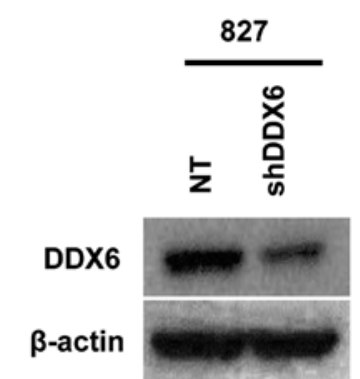

B

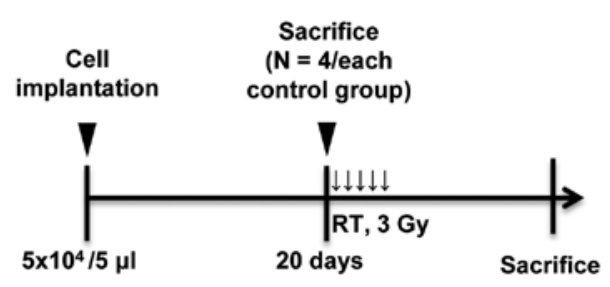

578
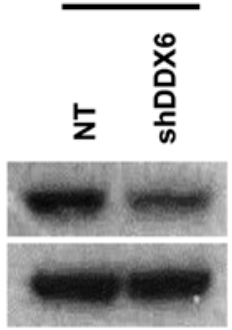

C
827 NT

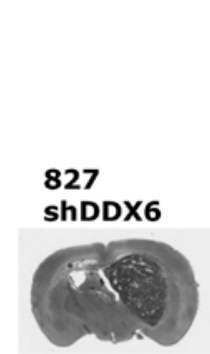

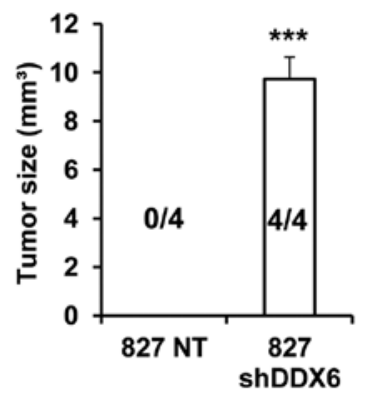

D $\quad$\begin{tabular}{ll}
-827 NT & $\ldots .8827$ shDDX6 \\
\hline--827 NT Radiation & --827 shDDX6 Radiation
\end{tabular}

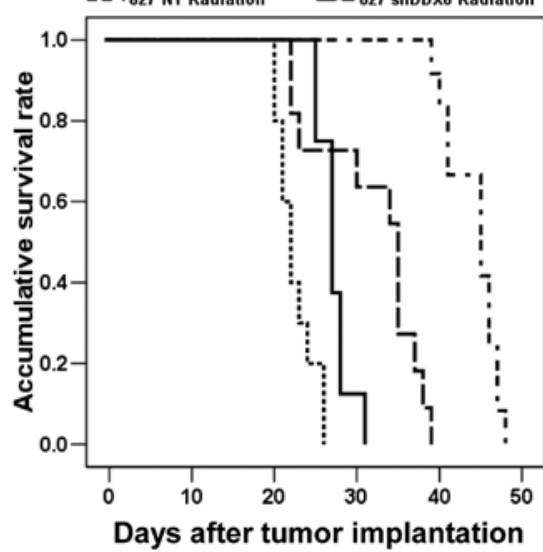

\begin{tabular}{|l|c|c|c|}
\hline \multicolumn{1}{|c|}{ Groups } & $\begin{array}{c}\text { Median } \\
\text { Survival } \\
\text { day }\end{array}$ & $\begin{array}{c}\text { ILS (\%, } \\
\text { vs } \\
\text { Group I) }\end{array}$ & $\begin{array}{c}p \text { value } \\
\text { (vs } \\
\text { Group I) }\end{array}$ \\
\hline I. 827 NT & 27 & - & - \\
\hline $\begin{array}{c}\text { II. } 827 \text { NT } \\
\text { w/ RT }\end{array}$ & 45 & $67 \%$ & $p<0.01$ \\
\hline $\begin{array}{l}\text { III. } 827 \text { shDDX6 } \\
\text { IV. } 827 \text { shDDX6 } \\
\text { w/ RT }\end{array}$ & 22 & $-19 \%$ & $p<0.01$ \\
\hline
\end{tabular}

Figure 2. Functional confirmation of decreased DDX6 in the xenograft model. (A) Animal experimental scheme for confirmation of radio-resistance in 827 shDDX6. (B) Clonogenicity improvement by decreased DDX6 expression identified in the 827 orthotopic xenograft model. (C,D) Survival graph in the 827 orthotopic xenograft model. shDDX6 cell implantation groups had decreased survival compared to non-targeted cell implantation groups against radiation treatment. \# ILS: increase of life span.

correct. The approach revealed the abundant expression of DDX6 in all treatment groups (Fig. 1C). These cells survived the radiation/TMZ treatment, which indicated DDX6 as a candidate gene regulating the resistance to radiation and/or TMZ treatment in GBM.

Functional confirmation of decreased DDX6 in the xenograft model. For functional validation of DDX6, a stable DDX6 knock-down cell line was established using lentiviral shRNA in two patient-derived cell lines (827 and 578). Since the 827 cells were resistant to TMZ treatment (Fig. 1B), TMZ sensitive cells were needed to confirm TMZ resistance in cells with abrogated expression of DDX6. Because 827 cells were used in shRNA screening and 578 cells were sensitive to TMZ treatment, these cell types were judged suitable. Analysis of protein level confirmed reduced DDX6 expression in both cell types in established DDX6 knock-down cells against non-target cells (Fig. 2A). Use of 827 cells allowed the establishment of a xenograft model that confirmed functional activity of DDX6 in radiation treatment, as evident in the first screening experiment (Fig. 2B). Non-targeted cells and shDDX6 cells were implanted in the mouse brain. Mice were sacrificed 20 days after cell implantation $(n=4$ in each control group) to verify tumor incidence. Tumor incidence and tumor size significantly improved $(\mathrm{P}<0.001)$ in the 827 shDDX6 cell implantation group compared to the NT cell group (Fig. 2C). No tumor mass was detected in NT implanted mouse brain. All shDDX6 implanted mice had tumor masses. Other mice were sacrificed when their body weight loss rate exceeded $20 \%$ or when abnormal behaviors (cachexia, lethargy and seizures) were displayed. The sacrifice day represented the survival end date for measuring median survival (Fig. 2D). The shDDX6s implanted mice (GIII) survived significantly shorter than NT implanted mice (GI) without radiation treatment. The median survival of all RT groups (NT-GII, shDDX6-GIV) was increased compared to untreated mice. The median survival 

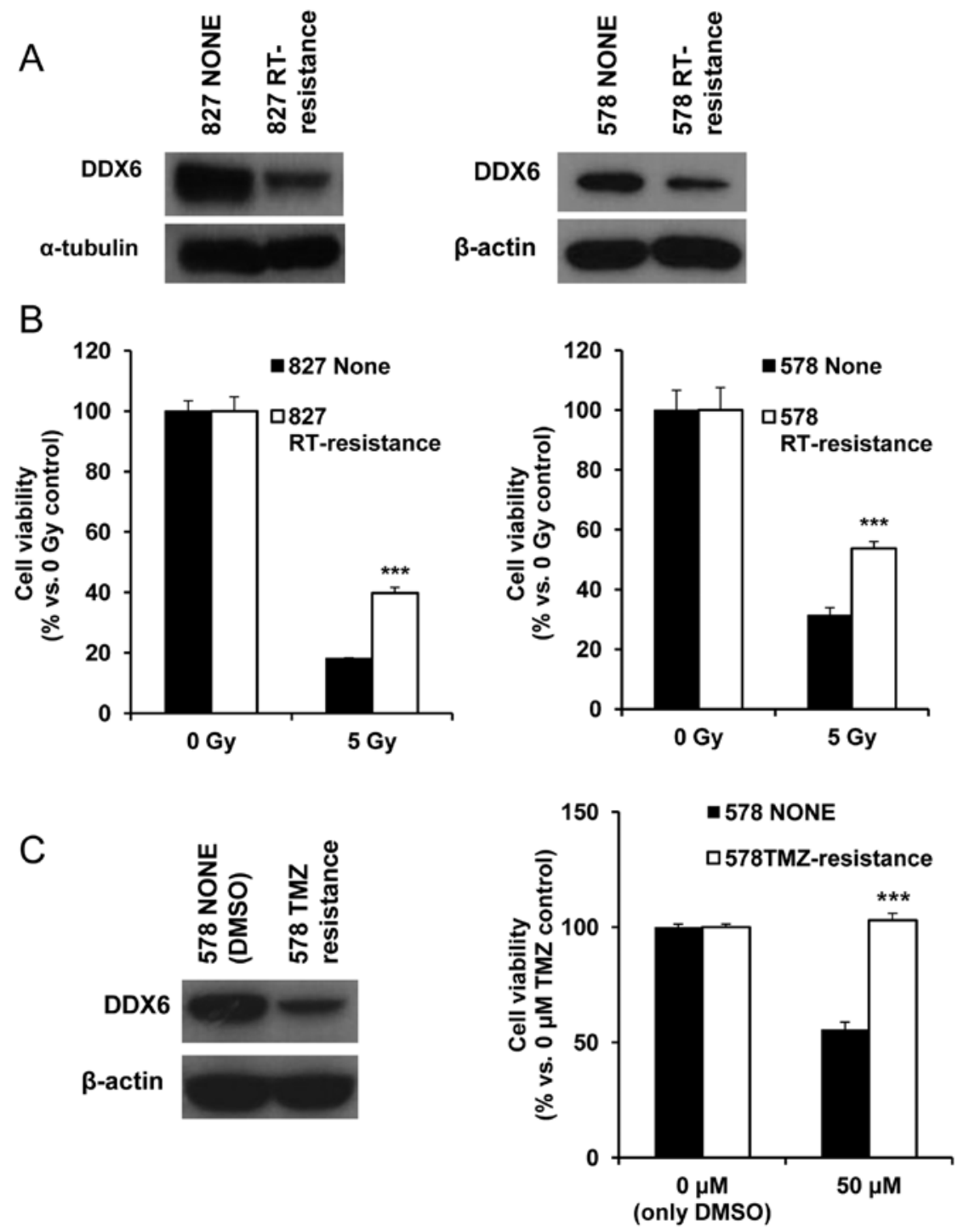

Figure 3. Establishment of RT/TMZ resistance cells to confirm alteration of DDX6 in RT/TMZ treatment. (A) DDX6 expression level of radio-resistant 827 and 578 cells. (B) Radiation response test for confirmation of radio-resistance. (C) TMZ response test to confirm TMZ resistance.

was slightly but significantly changed in radiation treated mice by decreased DDX6 (GII vs. GIV, P<0.01). Moreover, the range of survival end points within group was totally different between RT groups (GII, 39-48 days; GIV, 22-39 days). These results explained the earlier survival end point of the shDDX6 implanted mice compared to the NT group against RT. The results confirmed that decreased DDX6 increased tumorigenicity and induced a resistant response to radiation in vivo.

Establishment of RT/TMZ resistance cells to confirm alteration of DDX6 in RT/TMZ treatment. For confirmation of substantial DDX6 alteration against RT or TMZ treatment, we established RT/TMZ resistant cells by repeated treatment. Similar to DDX6 K/D cells, we used 827 and 578 patientderived cell types. Different protocols for the cell types reflected their different RT/TMZ sensitivity. The 827 cell type received 2 Gy radiation and the 578 cells received 1 Gy radiation to isolate $\mathrm{RT}$ resistant cells. The resistant response to radiation was confirmed by comparing post-treatment viability and altered DDX6 production (Fig. 3A and B). DDX6 protein expression decreased in both RT resistant cell types. Since 827 cells were resistant to TMZ treatment, TMZ sensitive cells were necessary to define TMZ resistance. In 578 cells, response to TMZ treatment was more sensitive and the response to RT was similar to that of 827 cells (data not shown; confirmed in Figs. 5A and 6A). Therefore, 578 cells were judged suitable to define TMZ resistance. These cells were treated with $5 \mu \mathrm{M} \mathrm{TMZ}$ and their viability compared to control 578 cells treated with DMSO, which was the TMZ solvent. Like radioresistant cells, TMZ resistant 578 cells also displayed diminished expression of DDX6 protein compared to control cells (Fig. 3C). This was evidence of decreased DDX6-induced resistance to radiation and TMZ.

Confirmation of tumor associated ability in 827 and 578 DDX6 knock-down cells. Next, alteration of tumor progression, proliferation and clonogenicity were assessed in DDX6 K/D cells. Although proliferation was unchanged, shDDX6s cells displayed significantly more frequent tumor initiation than non-target (NT) cells in both cell types (Fig. 4A and B). Since altered clonogenic potential had been demonstrated in the 827 xenograft model (Fig. 2C), the results suggested that 

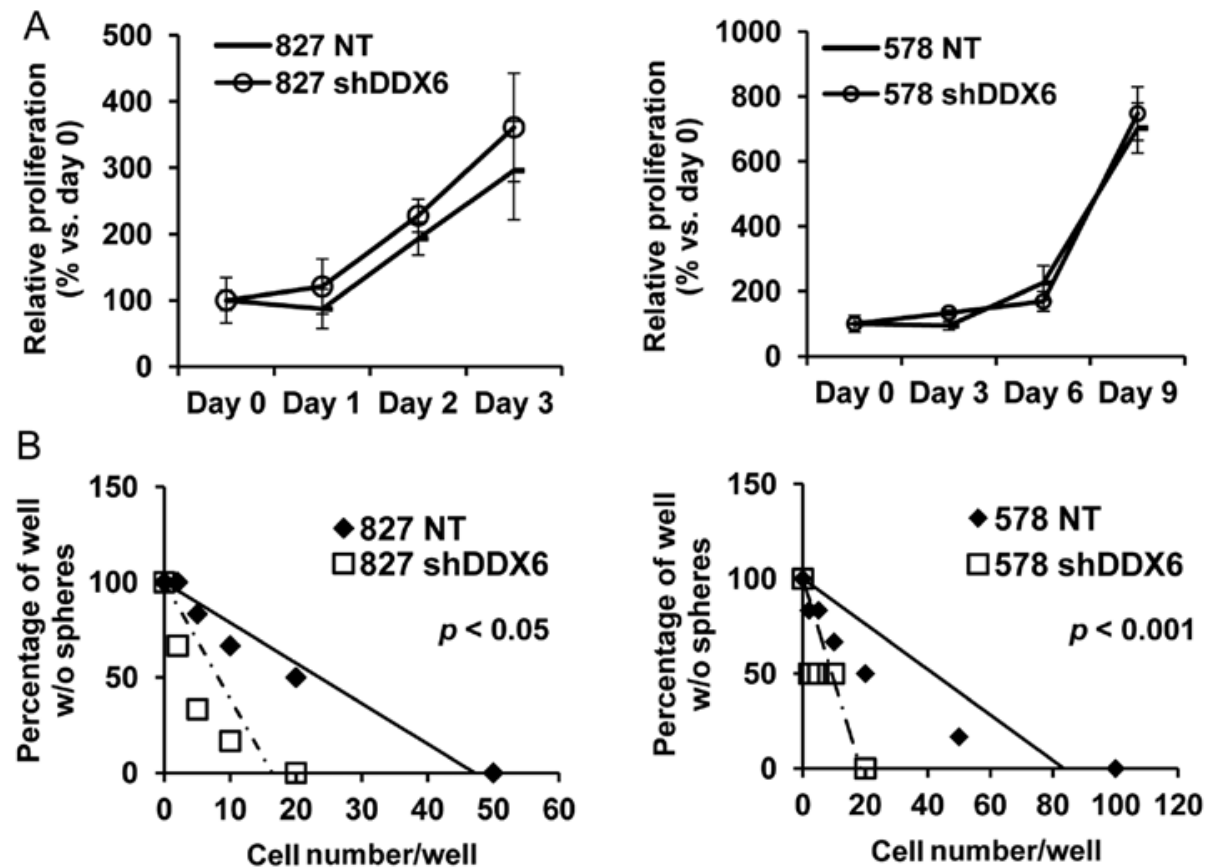

Figure 4. Confirmation of tumor associated ability in 827 and 578 DDX6 knock-down cells. (A) Proliferation assay in vitro. shDDX6 cells displayed no improved proliferation. (B) In vitro limiting dilution assay results. Sphere forming ability was significantly increased in cells with decreased DDX6 production.

A

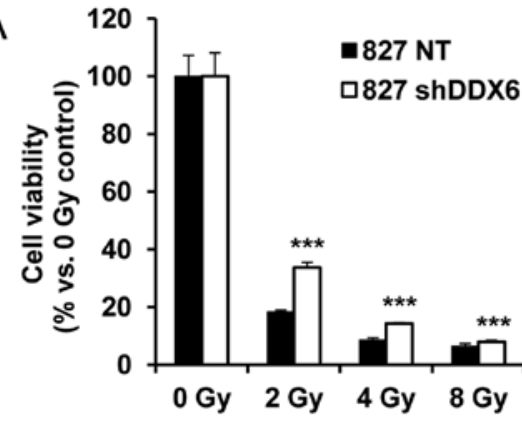

B

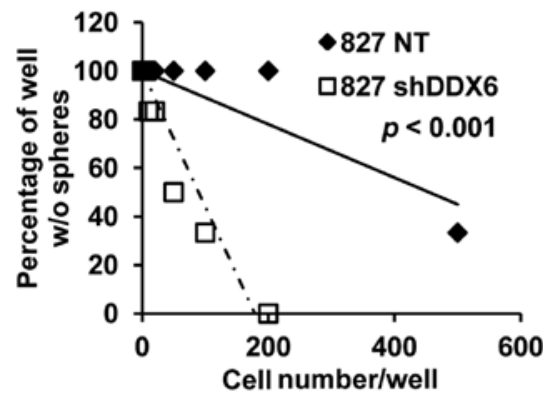

C

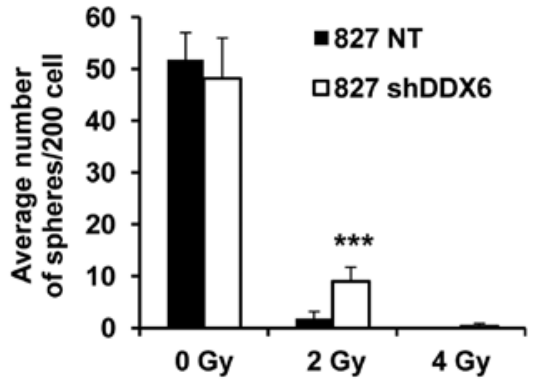

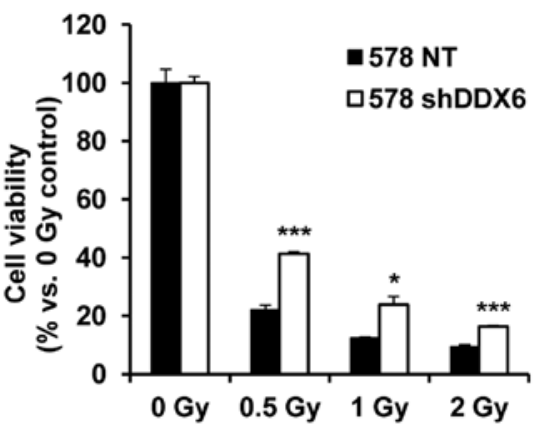
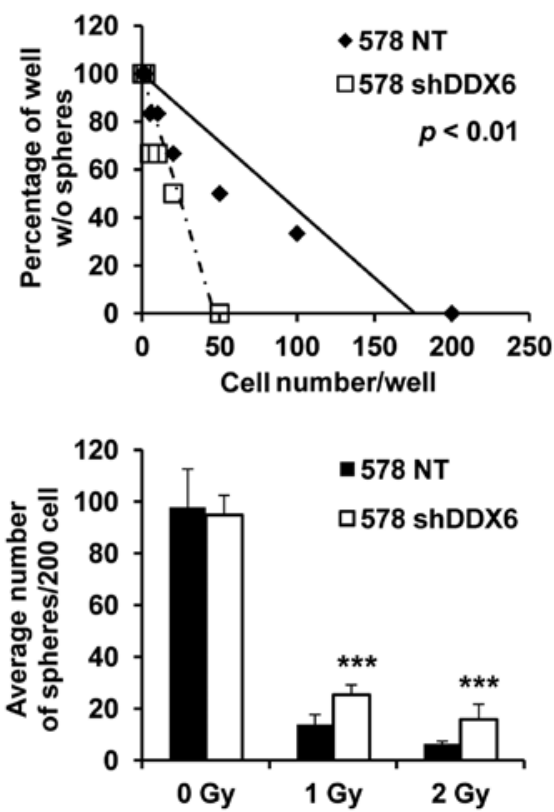

Figure 5. The cell decreased DDX6 expression acquired resistance against radiation treatment. (A) shDDX6 cells became resistant to radiation therapy. These results were retained while radiation dose increased. DDX6 expression level in the 827 and 578 cells. (B) Limiting dilution assay showed significantly. improved clonogenic ability by decreased DDX6 despite radiation treatment in both cells. The 827 cells treated with 2 Gy radiation and 578 cells treated with 1 Gy radiation. (C) Average of the generated sphere number increased by decreased DDX6 despite radiation treatment in both cells. These results were also retained while radiation dose increased. 

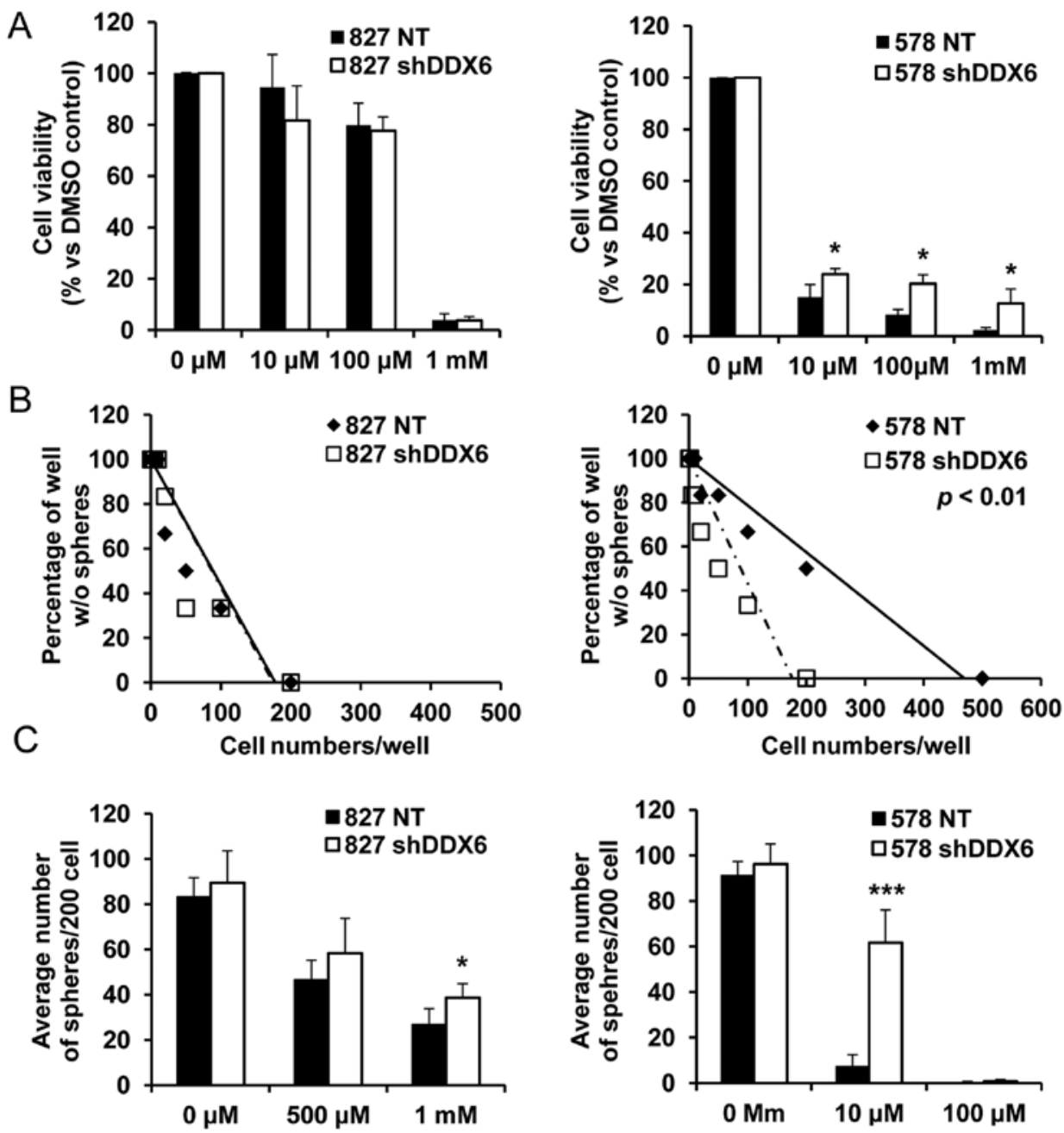

Figure 6. Decreased DDX6 expression influenced temozolomide resistance. (A) Temozolomide (TMZ) response was influenced by decreased DDX6. The 578 cells especially increased their viability against TMZ treatment by decreased DDX6, in the 827 cells it was not remarkable. (B) Limiting dilution assay against TMZ treatment. The 827 cells had no remarkable change in high TMZ concentration $(500 \mu \mathrm{M})$ and 578 cells had increased clonogenicity by decreased DDX6 against TMZ treatment $(10 \mu \mathrm{M})$. (C) Average of the generated sphere number against TMZ treatment. shDDX6 had more spheres against TMZ treatment especially in the 578 cells.

decreased DDX6 can influence the increased clonogenicity in vitro and in vivo.

Cell decreased DDX6 expression acquired resistance against radiation and temozolomide treatment. Irradiated 827 and 578 shDDX6s cells displayed significantly better survival than their corresponding NTs regardless of increased radiation intensity (Fig. 5A). Sphere formation was enhanced in untreated 827 and 578 shDDX6s cells. Therefore, clonogenic alteration due to the irradiation-mediated decreased DDX6 content was confirmed using two sphere formation assays (Fig. 5B and C). In the sphere limiting dilution assay (SLDA), irradiated shDDX6s formed spheres with fewer cells compared to NT 827 and 578 cells (Fig. 5B). Consistently, irradiated shDDX6s generated significantly more spheres than NT cells when cells were seeded with similar clonogenic potential (Fig. 5C). On the other hand, the observation that cells harboring shRNA targeting DDX6 were enriched after $\mathrm{TMZ}$ and combination treatment confirmed TMZ resistance due to decreased DDX6 expression. In an experiment with 827 cells, decreased DDX6 expression had no influence on cell viability and sphere forming ability after TMZ treatment (Fig. 6). Although the limiting dilution assay showed no influence by decreased DDX6 expression (Fig. 6B), average sphere numbers were slightly increased at a high concentration of TMZ (1 mM) with similar sphere forming capacity evident (Fig. 6C). Resistance to TMZ by decreased DDX6 was clearly evident in 578 cells. Viability and sphere forming ability were significantly improved after TMZ treatment in cells with decreased DDX6 expression (Fig. 6A and B), and the average sphere number with similar clonogenic capacity significantly increased in 578 shDDX6 cells, by $\sim 12$ times compared to NT cells (Fig. 6C).

Moreover, there were several primary-recurrent paired samples from the same patients in our patient sample bank, and several samples were cultured in vitro. As with shRNA screening (Fig. 1), we collected primary-recurrent paired samples to confirm the relationship between DDX6 expression and recurrence against CCRT including TMZ treatments. The DDX6 protein level was confirmed in two paired samples. In these samples, the DDX6 protein level of recurrent tumor cells were lower than in primary tumor 


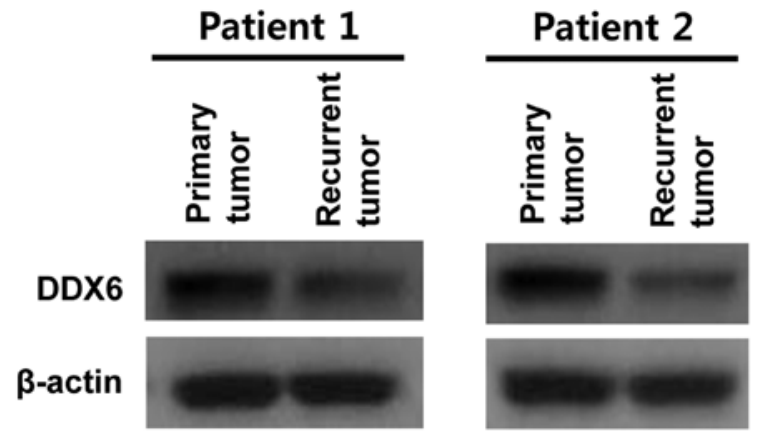

Figure 7. Alteration of DDX6 expression by CCRT between primaryrecurrent paired tumors. Protein level of DDX6 expression in paired tumor cells. Recurrent tumor cells had low DDX6 expression.

cells (Fig. 7). These results supported previous results using DDX6 K/D cells in which cells harboring shRNA survived better after radiation/TMZ treatment, and, in vivo, resistant or recurrent tumors remaining after anticancer therapy were associated with a poor DDX6 expression. All the above suggested that decreased DDX6 expression induces radiation/ $\mathrm{TMZ}$ resistance and influences prognosis.

\section{Discussion}

The present study was aimed at discovering potential genes involved in tumorigenic ability using shRNA screening techniques (14-16,23-25). Previous studies have utilized a pool of less than 1,000 shRNAs as it was difficult to select a single meaningful gene from a large-scale shRNA pool. Therefore, they conducted preliminary studies using a large-scale pool for more accurate results (26-28). Using a large-scale pool, they discovered a gene cluster that are involved in a particular pathway rather than identifying a single meaningful gene. In this study, we discovered DDX6 by treating shRNA-integrated cells with two different types of individual cellular stress despite using a large-scale pool.

Patient-derived xenograft models were used in our previously reported models and well representing tumor microenvironment and parental genomic characteristics compared to the in vitro models $(19,29,30)$. Because of its advantages, we performed shRNA screening using patient-derived xenograft models for identifying a particular gene responsible for CCRT resistance. Screening with approximately 8,000 shRNAs has previously identified several targets including transcriptional factors (RFX7 and ZNF 649), neurogenic factor (PRTG), tumor suppressor (BTG3), carcinogen (MYD88) and other cancer-related genes (31-36). Of those, 11 genes were selected in the treatment groups. Among them, BTG3 and MYD88 are known to be associated with cancer regulation and treatment resistance. Collectively, identification of the above genes supports the fidelity of our RNAi screen. Within the group, DDX6 was the only gene that was identified as a candidate hit in all three different treatment groups. Therefore, we sought to functionally validate DDX6.

DDX6 is an RNA helicase that regulates RNA modification (37). However, the functional activity of DDX6 remains unknown in cancer including GBM. Noteworthy, it has been reported that DDX6 upregulates tumorigenicity in colon cancer. Moreover, upregulation of DDX6 decreases miR-145 expression and induces tumorigenicity in $\operatorname{GBM}(38,39)$. Yet, our studies show that DDX6 induces RT/TMZ resistance rather than tumorigenic ability or miRNA modulation. Since DDX6 was the only gene that was identified in the treatmentresistant tumors and the results of our validation experiments were reliable, we suggest that DDX6 acts to regulate RT/ TMZ resistance in GBM. Although 827 DDX6 K/D cells showed unsettled response to TMZ, we reasoned that DDX6 protein level in 827 cells is lower than 578 cells and the level of 827 DDX6 K/D cells was not sufficient to present any phenotypic changes to TMZ resistance (data not shown). Therefore, we validated DDX6 function to TMZ resistance using 578 DDX6 K/D cells. Our data showed DDX6 playing a critical role in determining the sensitivity to CCRT in GBM patients. Therefore, DDX6 could be implemented as a therapeutic target in the clinic to predict a proficient response to CCRT treatment and to propose an alternative treatment.

To utilize DDX6 as a biomarker for patient prognosis, more evidence including functional activity of DDX6 to CCRT resistance need to be presented. Cancer stem cells (CSCs) are reported as vital factor for treatment resistance in cancer therapy (40-43). However, we found no reliable changes of CSC markers in our DDX6 K/D cells (data not shown, in $8 \mathrm{CSC}$ markers of GBM). Although we need more supplementary data for the mechanism of action, we consider miRNA could be involved in treatment resistance by DDX6 except CSC. miRNAs regulate many molecular actions in cells (44-46). In particular, several miRNAs have been reported for their role in treatment resistance. Reported miRNAs consists of miRNA-221/222, miRNA-181, miRNA-21, miRNA-195 (47-50). We need more studies for interaction between DDX6 and miRNAs to explain how DDX6 could regulate miRNAs in treatment resistance. Furthermore, DDX6 could be a putative tumor suppressor because of increased tumorigenicity of DDX6 K/D cells in vitro and in vivo. Further research using DDX6 overexpressed patient-derived cells is rneeded to confirm the tumor suppressor role of DDX6. Investigation of the roles of other candidate genes in response to CCRT resistance is also required.

\section{Acknowledgements}

This study was supported by a grant of the Korea Health Technology R\&D Project through the Korea Health Industry Development Institute (KHIDI), funded by the Ministry of Health and Welfare, Republic of Korea (HI14C3418) and the Global Frontier Project grant (NRF-2012M3A6A-2010-0029781) of National Research Foundation funded by the Ministry of Science, ICT and Future Planning (MSIP) of Korea.

\section{References}

1. Nishikawa R: Standard therapy for glioblastoma - a review of where we are. Neurol Med Chir (Tokyo) 50: 713-719, 2010.

2. Kong D-S, Kim ST, Kim E-H, Lim DH, Kim WS, Suh YL, Lee JI, Park K, Kim JH and Nam DH: Diagnostic dilemma of pseudoprogression in the treatment of newly diagnosed glioblastomas: The role of assessing relative cerebral blood flow volume and oxygen-6-methylguanine-DNA methyltransferase promoter methylation status. AJNR Am J Neuroradiol 32: 382-387, 2011. 
3. Locke VL, Davey RA and Davey MW: Modulation of drug and radiation resistance in small cell lung cancer cells by paclitaxel. Anticancer Drugs 14: 523-531, 2003.

4. Pires IM, Olcina MM, Anbalagan S, Pollard JR, Reaper PM, Charlton PA, McKenna WG and Hammond EM: Targeting radiation-resistant hypoxic tumour cells through ATR inhibition. Br J Cancer 107: 291-299, 2012.

5. Hegi ME, Liu L, Herman JG, Stupp R, Wick W, Weller M, Mehta MP and Gilbert MR: Correlation of O6-methylguanine methyltransferase (MGMT) promoter methylation with clinical outcomes in glioblastoma and clinical strategies to modulate MGMT activity. J Clin Oncol 26: 4189-4199, 2008.

6. Happold C, Roth P, Wick W, Schmidt N, Florea AM, Silginer M, Reifenberger G and Weller M: Distinct molecular mechanisms of acquired resistance to temozolomide in glioblastoma cells. J Neurochem 122: 444-455, 2012.

7. Goellner EM, Grimme B, Brown AR, Lin YC, Wang XH, Sugrue KF, Mitchell L, Trivedi RN, Tang JB and Sobol RW: Overcoming temozolomide resistance in glioblastoma via dual inhibition of $\mathrm{NAD}^{+}$biosynthesis and base excision repair. Cancer Res 71: 2308-2317, 2011.

8. Ramirez YP, Weatherbee JL, Wheelhouse RT and Ross AH: Glioblastoma multiforme therapy and mechanisms of resistance. Pharmaceuticals (Basel) 6: 1475-1506, 2013.

9. Hehlgans S and Cordes N: Caveolin-1: An essential modulator of cancer cell radio-and chemoresistance. Am J Cancer Res 1: 521-530, 2011

10. Kim Y, Kim KH, Lee J, Lee YA, Kim M, Lee SJ, Park K, Yang H, Jin J, Joo KM, et al: Wnt activation is implicated in glioblastoma radioresistance. Lab Invest 92: 466-473, 2012.

11. Fan Q-W and Weiss WA: Targeting the RTK-PI3K-mTOR axis in malignant glioma: Overcoming resistance. Curr Top Microbiol Immunol 347: 279-296, 2010.

12. Huang $\mathrm{H}$, Lin $\mathrm{H}$, Zhang $X$ and Li J: Resveratrol reverses temozolomide resistance by downregulation of MGMT in T98G glioblastoma cells by the NF-kB-dependent pathway. Oncol Rep 27: 2050-2056, 2012.

13. Lizardi PM, Forloni M and Wajapeyee N: Genome-wide approaches for cancer gene discovery. Trends Biotechnol 29: 558-568, 2011

14. Rudalska R, Dauch D, Longerich T, McJunkin K, Wuestefeld T, Kang TW, Hohmeyer A, Pesic M, Leibold J, von Thun A, et al: In vivo RNAi screening identifies a mechanism of sorafenib resistance in liver cancer. Nat Med 20: 1138-1146, 2014.

15. Xu H, Sun H, Zhang H, Liu J, Fan F, Li Y, Ning X, Sun Y, Dai S Liu B, et al: An shRNA based genetic screen identified Sesn2 as a potential tumor suppressor in lung cancer via suppression of Akt-mTOR-p70S6K signaling. PLoS One 10: e0124033, 2015.

16. Sroczynska P, Cruickshank VA, Bukowski J-P, Miyagi S, Bagger FO, Walfridsson J, Schuster MB, Porse B and Helin K shRNA screening identifies JMJD1C as being required for leukemia maintenance. Blood 123: 1870-1882, 2014.

17. Chen Y, Boland A, Kuzuoğlu-Öztürk D, Bawankar P, Loh B Chang CT, Weichenrieder O and Izaurralde E: A DDX6-CNOT1 complex and W-binding pockets in CNOT9 reveal direct links between miRNA target recognition and silencing. Mol Cell 54 737-750, 2014

18. Lee J, Kotliarova S, Kotliarov Y, Li A, Su Q, Donin NM, Pastorino S, Purow BW, Christopher N, Zhang W, et al: Tumor stem cells derived from glioblastomas cultured in bFGF and EGF more closely mirror the phenotype and genotype of primary tumors than do serum-cultured cell lines. Cancer Cell 9: 391-403, 2006.

19. Joo KM, Kim J, Jin J, Kim M, Seol HJ, Muradov J, Yang H, Choi YL, Park WY, Kong DS, et al: Patient-specific orthotopic glioblastoma xenograft models recapitulate the histopathology and biology of human glioblastomas in situ. Cell Rep 3: 260-273, 2013.

20. Rota LM, Lazzarino DA, Ziegler AN, LeRoith D and Wood TL: Determining mammosphere-forming potential: Application of the limiting dilution analysis. J Mammary Gland Biol Neoplasia 17: 119-123, 2012

21. Wei QCSL, Shen L, Zheng S and Zhu YL: Isolation and characterization of radiation-resistant lung cancer D6-R cell line. Biomed Environ Sci 21: 339-344, 2008.

22. Gomez-Casal R, Bhattacharya C, Ganesh N, Bailey L, Basse P, Gibson M, Epperly M and Levina V: Non-small cell lung cancer cells survived ionizing radiation treatment display cancer stem cell and epithelial-mesenchymal transition phenotypes. Mol Cancer 12: 94, 2013.
23. Mullenders $\mathbf{J}$ and Bernards R: Loss-of-function genetic screens as a tool to improve the diagnosis and treatment of cancer. Oncogene 28: 4409-4420, 2009.

24. Zhou P, Shaffer DR, Alvarez Arias DA, Nakazaki Y, Pos W, Torres AJ, Cremasco V, Dougan SK, Cowley GS, Elpek K, et al: In vivo discovery of immunotherapy targets in the tumour microenvironment. Nature 506: 52-57, 2014

25. Hitomi J, Christofferson DE, Ng A, Yao J, Degterev A, Xavier RJ and Yuan J: Identification of a molecular signaling network that regulates a cellular necrotic cell death pathway. Cell 135: 1311-1323, 2008

26. Wajapeyee N, Serra RW, Zhu X, Mahalingam M and Green MR: Oncogenic BRAF induces senescence and apoptosis through pathways mediated by the secreted protein IGFBP7. Cell 132: 363-374, 2008.

27. Chen S, Blank JL, Peters T, Liu XJ, Rappoli DM, Pickard MD, Menon S, Yu J, Driscoll DL, Lingaraj T, et al: Genome-wide siRNA screen for modulators of cell death induced by proteasome inhibitor bortezomib. Cancer Res 70: 4318-4326, 2010.

28. Mendes-Pereira AM, Sims D, Dexter T, Fenwick K, Assiotis I, Kozarewa I, Mitsopoulos C, Hakas J, Zvelebil M, Lord CJ, et al: Genome-wide functional screen identifies a compendium of genes affecting sensitivity to tamoxifen. Proc Natl Acad Sci USA 109: 2730-2735, 2012.

29. Cho YB, Hong HK, Choi Y-L, Oh E, Joo KM, Jin J, Nam DH, Ko YH and Lee WY: Colorectal cancer patient-derived xenografted tumors maintain characteristic features of the original tumors. J Surg Res 187: 502-509, 2014.

30. Lee HW, Lee JI, Lee SJ, Cho HJ, Song HJ, Jeong E, Seo YJ, Shin S, Joung JG, Kwon YJ, et al: Patient-derived xenografts from non-small cell lung cancer brain metastases are valuable translational platforms for the development of personalized targeted therapy. Clin Cancer Res 21: 1172-1182, 2015.

31. Amin S, Kumar A, Nilchi L, Wright K and Kozlowski M: Breast cancer cells proliferation is regulated by tyrosine phosphatase SHP1 through c-jun N-terminal kinase and cooperative induction of RFX-1 and AP-4 transcription factors. Mol Cancer Res 9: 1112-1125, 2011.

32. Severson PL, Tokar EJ, Vrba L, Waalkes MP and Futscher BW: Coordinate H3K9 and DNA methylation silencing of ZNFs in toxicant-induced malignant transformation. Epigenetics 8: 1080-1088, 2013.

33. Wong Y-H, Lu A-C, Wang Y-C, Cheng HC, Chang C, Chen PH, Yu JY and Fann MJ: Protogenin defines a transition stage during embryonic neurogenesis and prevents precocious neuronal differentiation. J Neurosci 30: 4428-4439, 2010.

34. Wang YC, Juan HC, Wong YH, Kuo WC, Lu YL, Lin SF, Lu CJ and Fann MJ: Protogenin prevents premature apoptosis of rostral cephalic neural crest cells by activating the $\alpha 5 \beta 1$-integrin. Cell Death Dis 4: e651, 2013.

35. Ou Y-H, Chung P-H, Hsu F-F, Sun T-P, Chang W-Y and Shieh S-Y: The candidate tumor suppressor BTG3 is a transcriptional target of p53 that inhibits E2F1. EMBO J 26: 3968-3980, 2007.

36. Kfoury A, Le Corf K, El Sabeh R, Journeaux A, Badran B, Hussein N, Lebecque S, Manié S, Renno T and Coste I: MyD88 in DNA repair and cancer cell resistance to genotoxic drugs. J Natl Cancer Inst 105: 937-946, 2013.

37. Weston A and Sommerville J: Xp54 and related (DDX6like) RNA helicases: Roles in messenger RNP assembly, translation regulation and RNA degradation. Nucleic Acids Res 34: 3082-3094, 2006.

38. Yang Y-P, Chien Y, Chiou G-Y, Cherng JY, Wang ML, Lo WL, Chang YL, Huang PI, Chen YW, Shih YH, et al: Inhibition of cancer stem cell-like properties and reduced chemoradioresistance of glioblastoma using microRNA145 with cationic polyurethane-short branch PEI. Biomaterials 33: 1462-1476, 2012.

39. Iio A, Takagi T, Miki K, Naoe T, Nakayama A and Akao Y: DDX6 post-transcriptionally down-regulates miR-143/145 expression through host gene NCR 143/145 in cancer cells. Biochim Biophys Acta 1829: 1102-1110, 2013

40. Dean M, Fojo T and Bates S: Tumour stem cells and drug resistance. Nat Rev Cancer 5: 275-284, 2005.

41. Baumann M, Krause M and Hill R: Exploring the role of cancer stem cells in radioresistance. Nat Rev Cancer 8: 545-554, 2008.

42. Rycaj K and Tang DG: Cancer stem cells and radioresistance. Int J Radiat Biol 90: 615-621, 2014.

43. Sotiropoulou PA, Christodoulou MS, Silvani A, Herold-Mende C and Passarella D: Chemical approaches to targeting drug resistance in cancer stem cells. Drug Discov Today 19: 1547-1562, 2014. 
44. Lu J, Getz G, Miska EA, Alvarez-Saavedra E, Lamb J, Peck D, Sweet-Cordero A, Ebert BL, Mak RH, Ferrando AA, et al: MicroRNA expression profiles classify human cancers. Nature 435: 834-838, 2005.

45. Hayes J, Peruzzi PP and Lawler S: MicroRNAs in cancer: Biomarkers, functions and therapy. Trends Mol Med 20: 460-469, 2014.

46. Lin S and Gregory RI: MicroRNA biogenesis pathways in cancer. Nat Rev Cancer 15: 321-333, 2015.

47. MillerTE, Ghoshal K, Ramaswamy B, Roy S, Datta J, Shapiro CL, Jacob S and Majumder S: MicroRNA-221/222 confers tamoxifen resistance in breast cancer by targeting p27Kip1. J Biol Chem 283: 29897-29903, 2008
48. Chen G, Zhu W, Shi D, Lv L, Zhang C, Liu P and $\mathrm{Hu}$ W: MicroRNA-181a sensitizes human malignant glioma U87MG cells to radiation by targeting Bcl-2. Oncol Rep 23: 997-1003, 2010.

49. Wong ST, Zhang XQ, Zhuang JT, Chan HL, Li CH and Leung GK: MicroRNA-21 inhibition enhances in vitro chemosensitivity of temozolomide-resistant glioblastoma cells. Anticancer Res 32: 2835-2841, 2012

50. Ujifuku K, Mitsutake N, Takakura S, Matsuse M, Saenko V, Suzuki K, Hayashi K, Matsuo T, Kamada K, Nagata I, et al: miR-195, miR-455-3p and miR-10a(*) are implicated in acquired temozolomide resistance in glioblastoma multiforme cells. Cancer Lett 296: 241-248, 2010. 